\title{
Diosmetin alleviates hypoxia-induced myocardial apoptosis by inducing autophagy through AMPK activation
}

\author{
QIJUN SI $^{1 *}$, YUJIE SHI $^{2 *}$, DANDAN HUANG ${ }^{3}$ and NA ZHANG \\ ${ }^{1}$ Clinical Laboratory, Zhuji People's Hospital, Zhuji, Zhejiang 311800; ${ }^{2}$ Cardiovascular Disease Institute, \\ PLA Army General Hospital, Beijing 100000; ${ }^{3}$ Preclinical School, North Sichuan Medical College, Nanchong, \\ Sichuan 637000; ${ }^{4}$ Department of Internal Medicine, The Hospital of Wuhan University, Wuhan, Hubei 430000, P.R. China
}

Received April 21, 2019; Accepted August 8, 2019

DOI: $10.3892 / \mathrm{mmr} .2020 .11241$

\begin{abstract}
Diosmetin has shown great potential in the control of several diseases. The aim of the present study was to evaluate the role of diosmetin as a candidate agent for the treatment of myocardial infarction which was mainly caused by hypoxia. The model of hypoxia-injured myocardial cells was established using the H9c2 cell line. Cell viability was determined using Cell Counting Kit-8, cell apoptosis was determined by Annexin V-FITC Apoptosis Detection Kit and cleaved caspase-3 level was assessed by western blot analysis. Autophagy was monitored using a commercial kit, and a well-established reporter system was used to confirm the role of diosmetin in autophagy. The activity of adenosine 5'-monophosphate-activated protein kinase (AMPK) signaling was detected by western blot analysis. Cell viability assay indicated that diosmetin alleviated hypoxia-induced cell death of H9c2 cells in a dose-dependent manner. Data of the apoptosis assay revealed that diosmetin reduced the proportion of apoptotic cells in the hypoxia-injured H9c2 cells. It was also found that the occurrence of autophagy was promoted when hypoxia-injured cells were treated with diosmetin alone, and results of the western blot analysis revealed that AMPK signaling was activated by diosmetin. Administration of diosmetin together with an inhibitor of autophagy (3-methyladenine, 3-MA) or AMPK (Compound C) was able to decrease the diosmetin-induced autophagy as well as the cytoprotective effects in the hypoxia-injured cells. Our study concluded that diosmetin exhibits a cytoprotective effect on hypoxia-injured myocardial cells by inducing autophagy and alleviating apoptosis. AMPK was demonstrated to regulate the observed effects
\end{abstract}

Correspondence to: Miss Na Zhang, Department of Internal Medicine, The Hospital of Wuhan University, 299 Bayi Road, Wuchang, Wuhan, Hubei 430000, P.R. China

E-mail: zhna_sc4223@tom.com

${ }^{*}$ Contributed equally

Key words: diosmetin, hypoxia, myocardial cells, apoptosis, autophagy, AMPK caused by diosmetin. This investigation confirmed diosmetin as a promising drug candidate for myocardial infarction treatment. The present findings regarding the inherent molecular mechanisms involved in the protective effects of diosmetin promote the clinical application of diosmetin.

\section{Introduction}

Myocardial infarction is defined worldwide as one of the most common cardiovascular diseases with an estimated number of cases of more than 600,000 in the US (1). A stress environment, such as oxidative stress and hypoxia, is thought to be a significant inducement for the irreversible damage to myocardial cells (2). Therefore, maintaining the normal function and prolonging the survival of myocardial cells are of great importance for the heart $(1,3)$. Hypoxia induces the death of myocardial cells mainly by triggering apoptosis (4-6). Strategies that can effectively suppress cell apoptosis alleviate hypoxia-induced cell death.

Diosmetin (3',5,7-trihydroxy-4'-methoxy flavone) is the aglycone of the flavonoid glycoside diosmin, which is abundant in Citrus species, olive leaves and spermine (7). Studies have demonstrated that diosmetin exhibits various medicinal properties including anticancer (8), anti-microbial (9), anti-inflammatory (10) and anti-oxidant (11) activities. Diosmetin was found to reduce secretion of TNF- $\alpha$, thus decreasing the inflammatory level, and the anti-oxidant effect by diosmetin was mainly ascribed to the upregulation of superoxide dismutase (11). The anticancer effect of diosmetin was reportedly related to its regulation of cell apoptosis (8). Studies of hepatocellular carcinoma and prostate cancer revealed that diosmetin functions as a tumor inhibitor mainly by inducing apoptosis $(8,12,13)$. However, the effect of diosmetin on apoptosis is not consistent. Zhang et al (14) documented that diosmetin suppresses neuronal apoptosis. Yet, the role of diosmetin in cardiomyocytes has not yet been investigated.

Autophagy is characterized as a survival mechanism that is responsible for removal of misfolded or wrongly assembled proteins, clearance of damaged organelles and elimination of intracellular pathogens (15). The relationship between autophagy and apoptosis has previously been discussed. Nikoletopoulou et al (16) summarized that autophagy and apoptosis may antagonize or assist each other, which is 
possibly attributed to the interplay among core factors involved in both processes (17). Autophagy is a process that can be accurately regulated by multiple signals including the nuclear factor (NF) $-\kappa \mathrm{B}$ pathway (10), the $\mathrm{p} 53 / \mathrm{Bcl}-2$ pathway (17) and BECN1/adenosine 5'-monophosphate-activated protein kinase (AMPK) $(18,19)$, which indicates an inconsistent role of autophagy in different diseases or diseases with different stages (15).

The present study was designed with an aim to explore the effects of diosmetin on hypoxia-injured myocardial cells. Subsequently, the potential involvement of autophagy in the diosmetin-mediated effects was focused on. Then the activity of AMPK signaling in diosmetin-treated cells was assessed to elucidate the intrinsic molecular mechanisms.

\section{Materials and methods}

Cell culture and treatment. The cardiomyocyte cell line $\mathrm{H} 9 \mathrm{c} 2$ derived from the rat was purchased from the American Type Culture Collection (ATCC, Manassas, VA, USA). Cells were maintained in DMEM (HyClone Laboratories Inc./GE Healthcare) as recommended. All medium used were supplemented with $10 \%$ fetal bovine serum (FBS; HyClone Laboratories Inc./GE Healthcare) and cells were routinely cultured in a humidified incubator at $37^{\circ} \mathrm{C}$ under $5 \% \mathrm{CO}_{2}$.

$\mathrm{H} 9 \mathrm{c} 2$ cells at $70-80 \%$ confluence were maintained in serum-free medium with low glucose for $12 \mathrm{~h}$ for cell starvation. Subsequently, 5, 10 or $15 \mu \mathrm{g} / \mathrm{ml}$ diosmetin (Selleck Chemicals) dissolved in DMSO, 5 mM 3-methyladenine (3-MA; Selleck Chemicals) dissolved in PBS or $10 \mu \mathrm{M}$ compound $\mathrm{C}$ (Selleck Chemicals) dissolved in DMSO were added to the cell cultures immediately after serum starvation. The cells were then cultured in normoxic conditions $\left(74 \% \mathrm{~N}_{2}, 5 \% \mathrm{CO}_{2}\right.$ and $\left.21 \% \mathrm{O}_{2}\right)$ for $1 \mathrm{~h}$ prior to being cultured in hypoxic conditions $\left(94 \% \mathrm{~N}_{2}\right.$, $5 \% \mathrm{CO}_{2}$ and $1 \% \mathrm{O}_{2}$ ) for $48 \mathrm{~h}$, a duration which was frequently used in several previous studies $(20,21)$. As the non-treated group (NT), cells were maintained in normoxic conditions for the duration of the experiment.

Cell viability assay. For determination of cell viability, H9c2 cells exposed to hypoxia or the non-treated group were plated into a flat bottom 96 -well plate at $4 \times 10^{3}$ cells per well in triplicate with $100 \mu \mathrm{l}$ medium. Ten microliters of Cell Counting Kit-8 reagent (CCK-8; Dojindo) was added into each well. After incubation for $2 \mathrm{~h}$, optical density (OD) value at the wavelength of $450 \mathrm{~nm}$ was measured using a microplate reader (Bio-Rad Laboratories, Inc.).

Cell apoptosis assay. Cell apoptosis was detected using the Annexin V-FITC Apoptosis Detection Kit (Calbiochem/EMD/Merck KGaA) according to the manufacturer's instructions.

Western blot analysis. Cell lysates were prepared using RIPA lysis buffer (Cell Signaling Technology) supplemented with protease inhibitor and phosphatase inhibitors for protein extraction. Concentration of total protein was determined by the Pierce Coomassie (Bradford) Protein Assay Kit (Thermo Fisher Scientific, Inc.). Then protein samples $(20 \mu \mathrm{g})$ were resolved on $8 \%$ SDS-PAGE and transferred to polyvinylidene difluoride membranes. The rinsed membranes were incubated with primary antibodies targeting cleaved caspase-3 (cat. no. 9654), phospho-AMPKa (cat. no. 2531), AMPK (cat. no. 5832), phospho-ULK1 (cat. no. ab229537), ULK1 (cat. no. 8054) or $\beta$-actin (cat. no. 4970 ) at $4^{\circ} \mathrm{C}$ overnight. After washing, the membrane was incubated with a secondary antibody conjugated with horseradish peroxidase (cat. no. 7074). The signals were developed using Super Signal West Dura Extended Duration chemiluminescence substrate (Thermo Fisher Scientific, Inc.) and measured by ChemiDoc ${ }^{\mathrm{TM}}$ XRS+ System (Bio-Rad Laboratories, Inc.). All primary antibodies and secondary antibodies were purchased from Cell Signaling Technology except for phospho-ULK1 antibody which was purchased from Abcam. All antibodies were used in a 1:1,000 dilution as recommended by the supplier.

Autophagy detection. Cell autophagy was detected using a CYTO-ID ${ }^{\circledR}$ Autophagy detection kit (Enzo Life Sciences) following the manufacturer's instructions. Briefly, H9c2 cells were harvested and washed with PBS. Cells were then stained with CYTO-ID green fluorescence regents for $30 \mathrm{~min}$ at $37^{\circ} \mathrm{C}$. After being washed with assay buffer provided in the CYTO-ID ${ }^{\circledR}$ Autophagy detection kit, cells were fixed with $4 \%$ paraformaldehyde in PBS for $20 \mathrm{~min}$. Cells labeled with fluorescence were analyzed by flow cytometry and photographed using a Leica DM6000B fluorescence microscope (Leica Microsystems, Inc.). Higher fluorescence intensity indicated a higher level of autophagy.

Autophagy LC3 HiBiT Reporter Assay. An Autophagy LC3 HiBiT Reporter Assay System (Promega) provides a quick, efficient and common method with which to assess the effects of compounds on autophagy. The effects of diosmetin on autophagy were confirmed using this assay. Briefly, 293T cells provided by this system were routinely cultured with DMEM supplemented with $10 \%$ FBS and $500 \mu \mathrm{g} / \mathrm{ml} \mathrm{G} 418$ (Selleck Chemicals). For the assays, $293 \mathrm{~T}$ cells were plated into an opaque, white tissue-culture 96 -well plate at $2 \times 10^{4}$ cells per well in triplicate. After incubation for $24 \mathrm{~h}$, the cells were treated with diosmetin at different concentrations (5, 10 and $15 \mu \mathrm{g} / \mathrm{ml})$; cells treated with an equal amount of solvent were as control. After incubation at $37^{\circ} \mathrm{C}$ for another $48 \mathrm{~h}$, the luminescence signal was detected with an EnVision 2105 Multimode Plate Reader (PerkinElmer) following the manufacturer's instructions. The luminescence signal of treated cells was normalized to the control. Three independent experiments were performed.

Statistical analysis. Statistical analysis was performed using SPSS 19.0 (SPSS, Inc.). Data are presented as a mean \pm standard deviation of three parallel experiments. One-way ANOVA analysis was used for comparison of more than two groups, followed by Newman-Keuls post hoc test. A P-value $<0.05$ was considered to indicate a statistically significant difference.

\section{Results}

Diosmetin alleviates hypoxia-induced myocardial apoptosis. To ascertain the effects of diosmetin on hypoxia-induced injury in cardiomyoblasts, H9c2 cells were treated with 
A

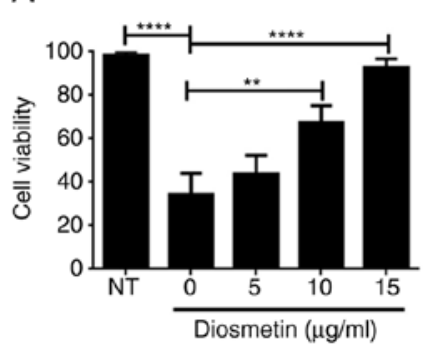

$\mathrm{D}$

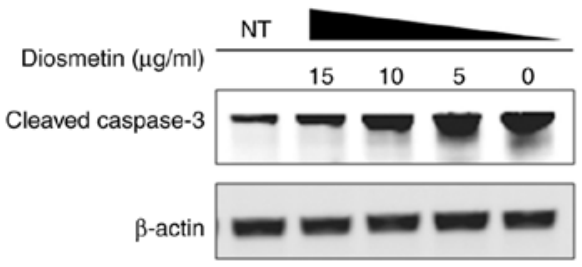

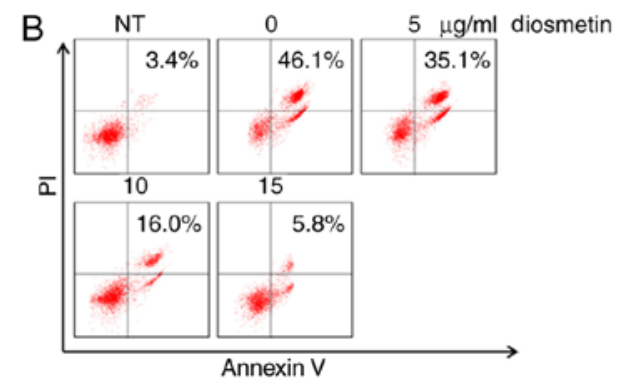

C

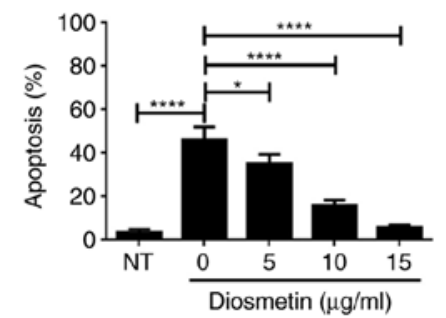

E

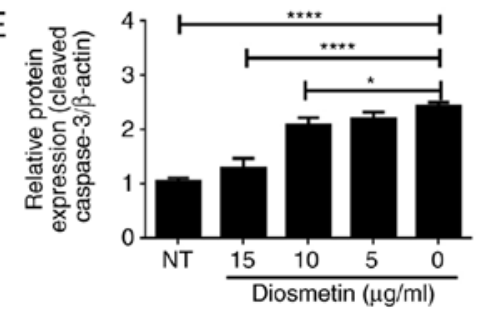

Figure 1. Effects of diosmetin on the $\mathrm{H} 9 \mathrm{c} 2$ cell line. (A) Cells were treated with diosmetin at different concentrations $(0,5,10$ and $15 \mu \mathrm{g} / \mathrm{ml}) \mathrm{prior}$ to being cultured in a hypoxic condition. Cells constantly cultured in a normoxic condition were considered as the non-treated group (NT). Cell viability was determined using CCK-8 assay. (B and C) Cell apoptosis was detected using a Annexin V-FITC Apoptosis Detection Kit. Double-staining cells were defined as apoptotic cells. (D and E) Cleaved caspase-3, an apoptosis-related protein, was assessed using western blot analysis. Three independent experiments were conducted for all assays. ${ }^{*} \mathrm{P}<0.05,{ }^{* *} \mathrm{P}<0.01,{ }^{* * * *} \mathrm{P}<0.0001$.

gradient dilutions of diosmetin $(5,10,15 \mu \mathrm{g} / \mathrm{ml})$ before exposure to hypoxia. Results of the CCK- 8 assay indicated that cell viability was significantly lower in the cells exposed to hypoxia when compared to the cells not exposed to hypoxia (NT) (Fig. 1A). It was also observed that cell viability was obviously higher in the cells treated with 10 and $15 \mu \mathrm{g} / \mathrm{ml}$ diosmetin than that in cells exposed only to hypoxia among all hypoxia-treated groups (Fig. 1A). These data demonstrated that exposure to hypoxia decreased cell survival, while diosmetin protected $\mathrm{H} 9 \mathrm{c} 2$ cells from hypoxia-induced decrease in cell survival in a dose-dependent manner. Furthermore, cell apoptosis was detected by PI/Annexin V staining assay. The results revealed that exposure to hypoxia significantly induced H9c2 apoptosis, resulting in considerable double-labeled population (46.1\%, Fig. 1B). Notably, the double-labeled apoptotic population was $35.1,16.0$ and 5.8 in the cells pretreated with 5,10 and $15 \mu \mathrm{g} / \mathrm{ml}$ diosmetin, respectively (Fig. 1B and C). Moreover, western blot analysis showed that hypoxia upregulated expression of cleaved caspase-3, while the upregulated cleaved caspase- 3 was effectively suppressed when cells were treated with diosmetin at increasing concentrations (Fig. 1D and E). Collectively, diosmetin may protect H9c2 cells from hypoxia-induced cell death via alleviating hypoxia-induced cell apoptosis in a dose-dependent manner.

Cell autophagy induced by diosmetin. The effects of diosmetin on cell autophagy were also investigated in the present study. CYTO-ID ${ }^{\circledR}$ Autophagy detection kit was used to detect cell autophagy, providing a rapid, specific and quantitative approach for monitoring autophagy. As shown in Fig. 2A-C, exposure to hypoxia induced slight cell autophagy which was subsequently strengthened by additional treatment of diosmetin in a dose-dependent manner. An Autophagy LC3 HiBiT Reporter Assay System was used to investigate whether diosmetin functions as an autophagy inducer or inhibitor. Currently, the direct effects of diosmetin on cell autophagy were estimated using this reporter assay system. The results indicated that luminescence intensity was comparably lower in cells which were treated with 10 and $15 \mu \mathrm{g} / \mathrm{ml}$ diosmetin (Fig. 2D), confirming the role of diosmetin as an autophagy inducer in a dose-dependent manner.

Autophagy inhibition by 3-methyladenine neutralizes the protective effects of diosmetin on hypoxia-injured cells. 3-Methyladenine (3-MA) is a well-studied autophagy inhibitor. 3-MA was used as a cell autophagy inhibitor to confirm whether diosmetin-induced autophagy is essential in maintaining its protective effect on cell survival. Results of the CCK- 8 assay illustrated that diosmetin treatment significantly enhanced the cell viability of the hypoxia-injured cells, which was previously evidenced. Subsequently, it was demonstrated that an additional treatment of 3-MA observably decreased cell viability in the hypoxia-injured cells treated with diosmetin (Fig. 3A). It can be concluded that the protective effects of diosmetin on hypoxia-injured cells were suppressed by autophagy inhibition; namely, autophagy is an indispensable mechanism that mediates the protection of diosmetin in regards to cell proliferation. apoptosis analysis also revealed that 3-MA partially reversed apoptosis inhibition induced by diosmetin (Fig. 3B and C). Cleaved caspase-3 was also upregulated in cells treated with diosmetin and 3-MA as compared to cells only treated with diosmetin (Fig. 3D and E). The above findings imply that autophagy is the main mechanism responsible for diosmetin-induced proliferation protection.

Activation of AMPK by diosmetin results in induction of autophagy and inhibition of apoptosis. Activation of Unc-51 like autophagy activating kinase 1 (ULK1), a kinase that is importantly involved in cell autophagy, can be regulated by adenosine 5'-monophosphate-activated protein kinase (AMPK) in a direct or even an indirect manner (19). Therefore, 
A

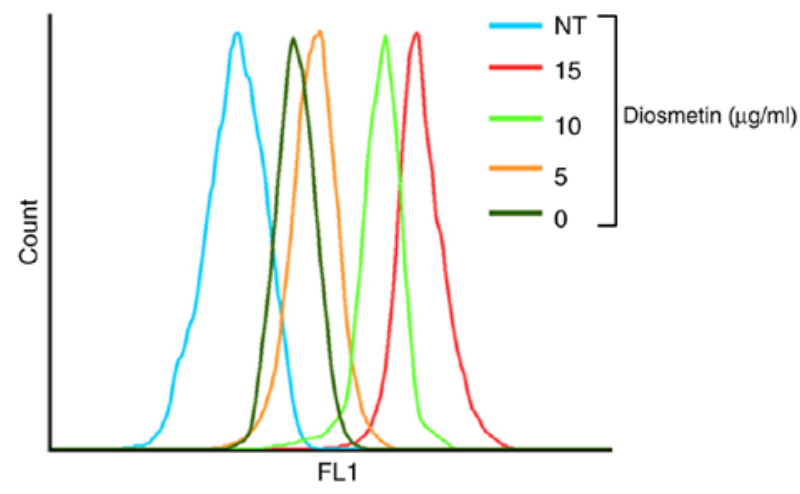

C

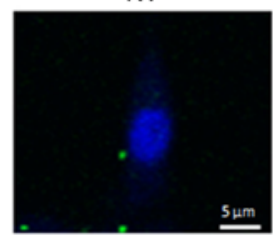

10

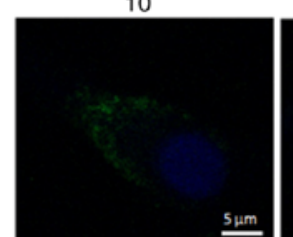

0

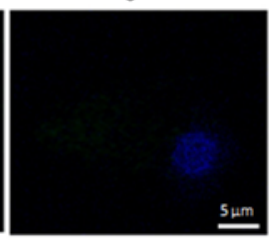

15

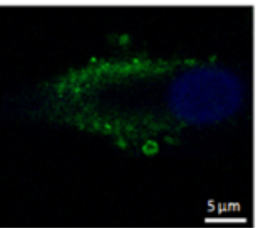

B

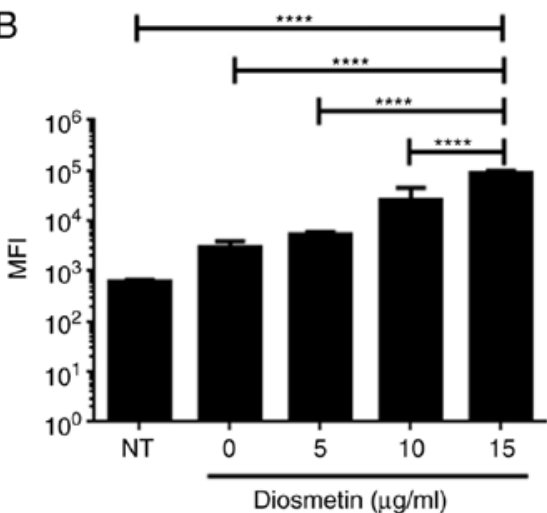

5

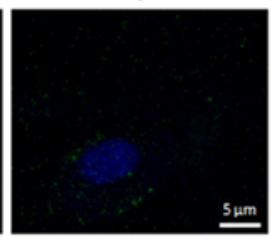

$\underline{5 \mu m}$

D

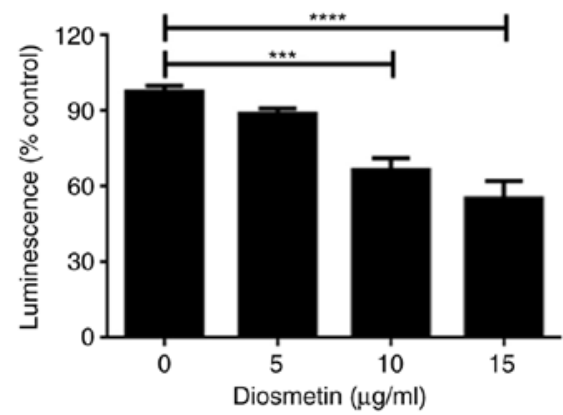

Figure 2. Diosmetin functions as an autophagy inducer. (A and B) The occurrence of autophagy was detected using a CYTO-ID ${ }^{\circledR}$ Autophagy detection kit in hypoxia-injured cells previously treated with diosmetin. Median fluorescence intensity (MFI) of channel FL1 (green fluorescence) was assessed using flow cytometry, and the higher fluorescence intensity represented stronger autophagy. (C) Images were captured using a fluorescence microscope; blue fluorescence represents Hoechst 33342 staining of cell nuclei and green fluorescence indicates CYTO-ID green staining of autophagic vesicles. (D) An independent Autophagy LC3 HiBiT Reporter Assay System based on 293T cells was performed to verify the role of diosmetin in autophagy; a lower luminescence signal represents stronger autophagy. At least six independent experiments were conducted for above assays. ${ }^{* * *} \mathrm{P}<0.001,{ }^{* * * * *} \mathrm{P}<0.0001$. To note, cells constantly cultured in a normoxic condition were considered as the non-treated group (NT).

A

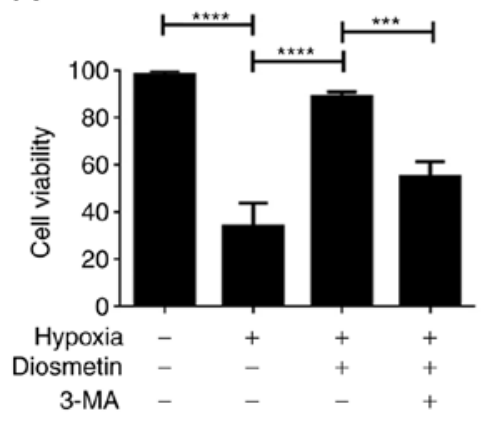

$B$

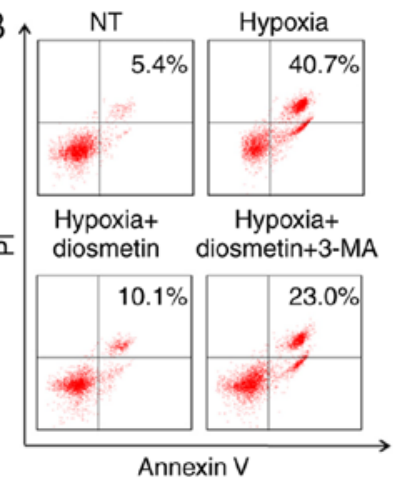

C

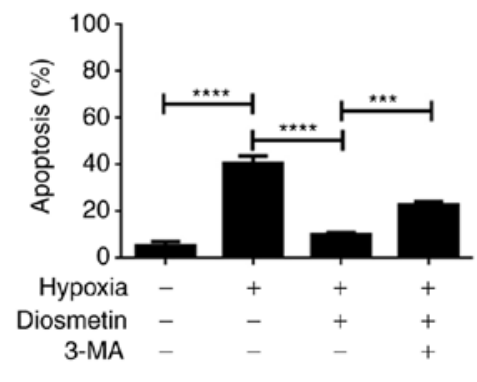

D

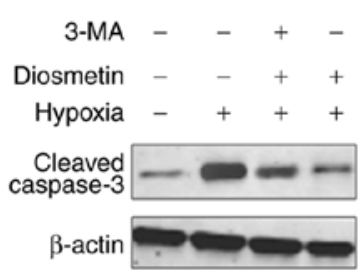

$\mathrm{E}$

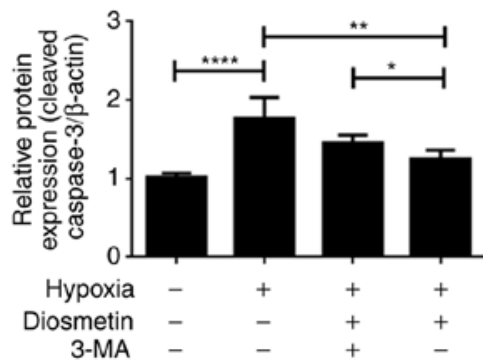

Figure 3. Induction of autophagy is essential for the cytoprotective effects of diosmetin on hypoxia-injured cells. (A) Cells were treated with diosmetin alone or together with 3-MA prior to being cultured in a hypoxic condition. The cells constantly cultured in a normoxic condition were considered as the non-treated group (NT). Cell viability was determined using the CCK-8 assay. (B and C) Cell apoptosis was detected using the Annexin V-FITC Apoptosis Detection Kit. The double-staining cells were defined as apoptotic cells. (D and E) Cleaved caspase-3 was assessed by western blot analysis. Three independent experiments were conducted for all assays. ${ }^{*} \mathrm{P}<0.05,{ }^{* *} \mathrm{P}<0.01{ }^{* * *} \mathrm{P}<0.001,{ }^{* * * * *} \mathrm{P}<0.0001$. 
A

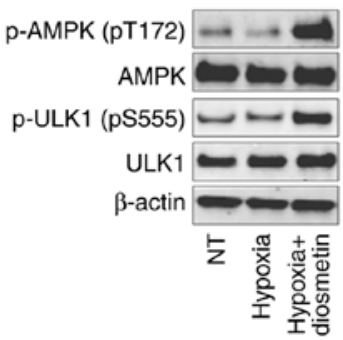

B

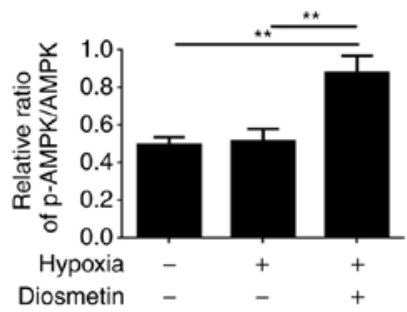

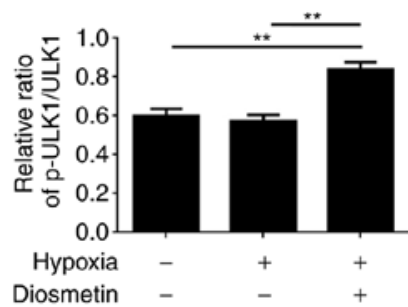

C
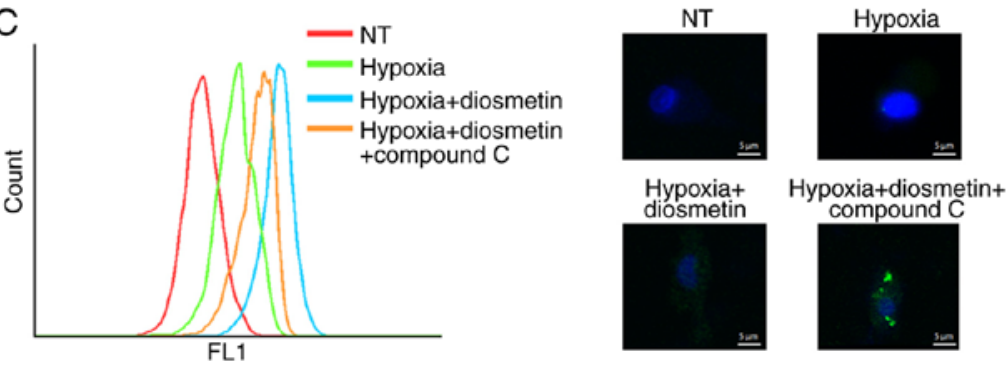

D

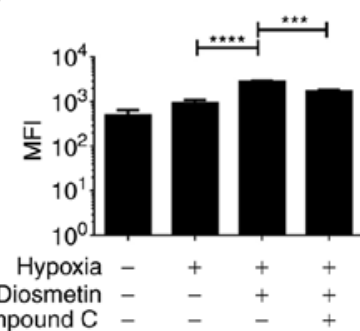

E

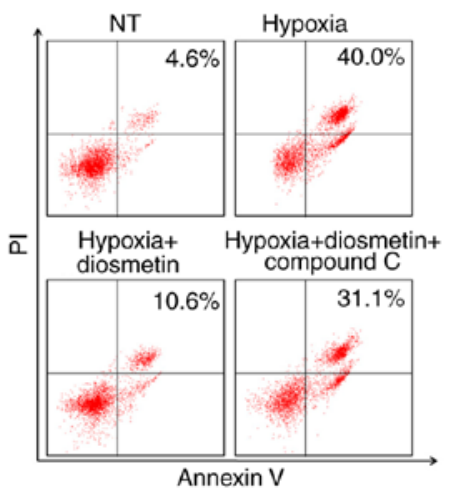

F

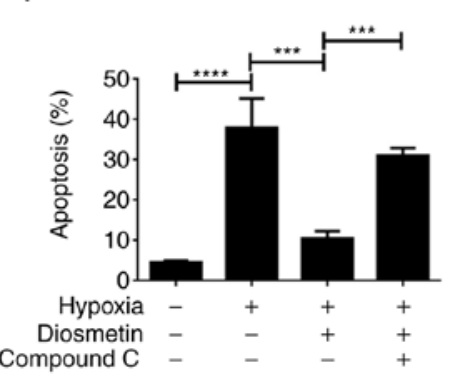

Figure 4. AMPK is involved in the regulatory effects of diosmetin on autophagy and apoptosis. (A and B) Expression of AMPK and ULK1 as well as their phosphorylation levels (p-AMPK and p-ULK1) were detected by western blot analysis in diosmetin-treated and hypoxia-exposed cells. (C and D) Cells were treated with diosmetin alone or together with compound C prior to being cultured in a hypoxic condition. Autophagy was monitored using a CYTO-ID ${ }^{\circledR}$ Autophagy detection kit by flow cytometry and fluorescence microscopy. Blue fluorescence represents Hoechst 33342 staining of cell nuclei and green fluorescence indicates CYTO-ID green staining of autophagic vesicles. (E and F) Cell apoptosis was detected using the Annexin V-FITC Apoptosis Detection Kit. Three independent experiments were conducted for all assays. ${ }^{* *} \mathrm{P}<0.01,{ }^{* * *} \mathrm{P}<0.001,{ }^{* * * *} \mathrm{P}<0.0001$. To note, cells constantly cultured in a normoxic condition were considered as the non-treated group (NT). AMPK, adenosine 5'-monophosphate-activated protein kinase; ULK1, Unc-51 like autophagy activating kinase 1.

we aimed to ascertain whether the AMPK signaling pathway is involved in diosmetin-induced autophagy and apoptosis inhibition. Phosphorylated (p)-AMPK, AMPK, p-ULK1 and ULK1 were detected by western blot analysis. The results indicated that the levels of p-AMPK and p-ULK1 in hypoxia-injured cells were significantly increased by diosmetin (Fig. 4A and B). Compound C, an effective and widely used AMPK inhibitor, was used together with diosmetin in cells before hypoxia treatment. Cell autophagy was detected using CYTO-ID ${ }^{\circledR}$ Autophagy detection kit in hypoxia-injured cells treated with diosmetin alone or combined with compound $\mathrm{C}$. It was found that additional administration of compound $\mathrm{C}$ indeed significantly attenuated autophagy as compared to treatment with diosmetin alone (Fig. 4C and D). PI/Annexin V staining assay also showed that the apoptotic population in the hypoxia-injured cells treated with diosmetin was increased from 10.6 to $31.1 \%$ when compound $\mathrm{C}$ was additionally applied (Fig. 4E and F). Based on the observed results, it was proposed that diosmetin-induced autophagy and apoptosis inhibition were partially mediated by activation of AMPK.

\section{Discussion}

Myocardial infarction is globally recognized as one of the most common cardiovascular diseases consistently threatening human health (3). Despite the fact that various advanced strategies have been developed, myocardial infarction is still diagnosed with a continually increasing incidence and high mortality rate $(22,23)$. Thus, effective treatment strategies are urgently needed. Recent studies have established that myocardial infarction is mainly caused by ischemia or hypoxia which may further result in myocardial cell apoptosis $(2,24)$. Based on these mechanistic findings, studies have reported several compounds as potential and promising candidates for myocardial infarction treatment. Canstatin and atorvastatin were respectively found to improve myocardial infarction via attenuation of cell apoptosis $(3,25)$. Diosmetin has recently received extensive concerns for its anti-oxidative, anti-inflammatory and anti-apoptotic effects in various diseases $(8,10,11,26)$. Hence, diosmetin was investigated as a potential candidate for improvement of myocardial infarction caused by hypoxia. 
The results of the present study revealed that diosmetin efficiently protected and increased the cell viability of $\mathrm{H} 9 \mathrm{c} 2$ cells by inhibiting apoptosis indicated by decreased cleaved caspase-3. It was further found that diosmetin promoted cell autophagy, although hypoxia induced slight autophagy. Autophagy is characterized as a conserved process involving the degradation of cytoplasmic components (15). Previous research has demonstrated a significant relationship between cell autophagy and apoptosis. Zhang et al (27) reported that hypoxia-induced apoptosis is negatively correlated with autophagy. Another investigation demonstrated that attenuation of hypoxia-induced cardiomyocyte apoptosis by overexpressed BAG3 was mainly ascribed to induction of autophagy (21). To ascertain whether induction of autophagy is indispensable for diosmetin-associated protection from apoptosis, 3-MA, an autophagy inhibitor, was used to suppress autophagy. Similar to previous studies $(21,27,28)$, the present results revealed that inhibition of autophagy indeed compromised the protective effects of diosmetin, indicating an essential role of autophagy in the diosmetin-associated protection from apoptosis. The role of autophagy, described as a double-edged sword, has been recently proposed in several reviews which explained that autophagy may also lead to cell death under certain conditions $(16,17)$. Thus, it can be seen that the interplay between autophagy and apoptosis is more complex than imaged and is of great significance for cell fate decision when facing stress conditions. In the present study, a hypoxia duration of $48 \mathrm{~h}$ was used to successfully induce cell death. However, the determination of autophagy and apoptosis in cells treated with hypoxia for different durations is of great significance for a full understanding of autophagy and apoptosis under hypoxia.

ULK1, a protein kinase, is required for autophagy and has been reported as a substrate of adenosine monophosphate-activated protein kinase (AMPK) (29). AMPK is frequently known as a sensor that can be activated in response to environmental stress like hypoxia $(19,30)$. A previous study described that AMPK is positively involved in mitophagy regulation under chronic hypoxia (31). In the present study, it was found that AMPK was activated in cells receiving diosmetin prior to exposure to hypoxia. Appropriately, we hypothesized that diosmetin-induced autophagy is mediated by the AMPK/ULK1 signaling pathway. Therefore, an AMPK inhibitor (compound $C$ ) was used to evaluate the role of AMPK in diosmetin-induced autophagy. As expected, compound $\mathrm{C}$ treatment suppressed diosmetin-induced autophagy as well as the inhibitive effects on apoptosis. In fact, research demonstrated that single exposure to hypoxia was able to activate AMPK in H9c2 cells (31); however, this was not observed in our study. The different observations might be attributed to the slightly discrepant methods of hypoxia exposure in the two articles. Even so, slight autophagy was currently observed in cells exposed to hypoxia only. Presumably, autophagy as a protective mechanism is synchronously induced when cells are exposed to hypoxia. While ULK1 is not the only autophagy-associated protein that can be regulated by the AMPK signaling pathway. Beclin1, which plays a vital role in autophagy nucleation and elongation, was found to be regulated by AMPK (18). Diosmetin has been demonstrated to influence activation of the PI3K/Akt and NF- $\kappa \mathrm{B}$ signaling pathways $(10,14,32)$. The present study is the first to report that diosmetin is involved in regulation of the AMPK signaling pathway.

The in vitro evaluation of diosmetin as a promising drug candidate has been conducted in several diseases, among which diosmetin may function as an inhibitor of inflammation, an inducer or an inhibitor of apoptosis $(11,14,26,32)$. Existing research suggests that diosmetin may ameliorate the severity of chronic asthma and pancreatitis by anti-inflammatory action (10,33). Investigations of the effects of diosmetin on several types of cancer suggest that diosmetin inhibits the proliferation of prostate cancer and hepatoma cells via induction of apoptosis and cell cycle arrest $(8,12,13)$. A study assessed the effects of diosmetin on kidney injury and revealed that diosmetin was able to prevent ischemia-induced acute kidney injury via restraining apoptosis (34). Meanwhile, our present study was the first to investigate diosmetin in light of its protective role in promoting cardiomyocyte survival under a hypoxic environment. To date, diosmetin is a candidate agent with high potential in disease treatment. However, systemic and detailed research regarding the mechanisms related to its observed effects is limited. Apoptosis is not the only mechanism that may lead to hypoxia-induced cell death (16); other mechanisms will be given advanced attention in future investigations by our group. Mitochondrial membrane potential, oxygen consumption rate, endoplasmic reticulum stress, ATP generation rate and glycolysis (16) are suitable methods with which to comprehensively ascertain the mechanisms mediating diosmetin-associated protection of myocardial cell survival.

In conclusion, the present study revealed that diosmetin may protect myocardial cells from hypoxia-mediated cell death by inducing autophagy. Further investigation demonstrated that AMPK is involved in the regulation of diosmetin-induced autophagy. This study suggests diosmetin as a drug candidate for myocardial infarction treatment, and the results regarding AMPK contribute to the clinical application of diosmetin.

\section{Acknowledgements}

Not applicable.

\section{Funding}

No funding was received.

\section{Availability of data and materials}

The datasets used and/analyzed during the current study are available from the corresponding author on reasonable request.

\section{Authors' contributions}

NZ was involved in the conception and design of the study. QS, YS and DH were responsible for the data acquisition, analysis and interpretation. QS wrote the manuscript.

\section{Ethics approval and consent to participate}

Not applicable. 


\section{Patient consent for publication}

Not applicable.

\section{Competing interests}

The authors declare that they have no competing interests.

\section{References}

1. Li R, Geng HH, Xiao J, Qin XT, Wang F, Xing JH, Xia YF, Mao Y, Liang JW and Ji XP: miR-7a/b attenuates post-myocardial infarction remodeling and protects $\mathrm{H} 9 \mathrm{c} 2$ cardiomyoblast against hypoxia-induced apoptosis involving Sp1 and PARP-1. Sci Rep 6: 29082, 2016.

2. Huang Y, Chen JB, Yang B, Shen H, Liang JJ and Luo Q: RhoA/ROCK pathway regulates hypoxia-induced myocardial cell apoptosis. Asian Pac J Trop Med 7: 884-888, 2014.

3. Cheng WP, Lo HM, Wang BW, Chua SK, Lu MJ and Shyu KG: Atorvastatin alleviates cardiomyocyte apoptosis by suppressing TRB3 induced by acute myocardial infarction and hypoxia. J Formos Med Assoc 116: 388-397, 2017.

4. Liu B, Che W, Xue J, Zheng C, Tang K, Zhang J, Wen J and Xu Y: SIRT4 prevents hypoxia-induced apoptosis in H9c2 cardiomyoblast cells. Cell Physiol Biochem 32: 655-662, 2013.

5. Luo S, Gu X, Ma F, Liu C, Shen Y, Ge R and Zhu Y: ZYZ451 protects cardiomyocytes from hypoxia-induced apoptosis via enhancing MnSOD and STAT3 interaction. Free Radic Biol Med 92: 1-14, 2016.

6. Ashok A, Kanwar JR, Krishnan UM and Kanwar RK SurR9C84A protects and recovers human cardiomyocytes from hypoxia induced apoptosis. Exp Cell Res 350: 19-31, 2017.

7. Patel K, Gadewar M, Tahilyani V and Patel DK: A review on pharmacological and analytical aspects of diosmetin: A concise report. Chin J Integr Med 19: 792-800, 2013.

8. Oak C, Khalifa AO, Isali I, Bhaskaran N, Walker E and Shukla S: Diosmetin suppresses human prostate cancer cell proliferation through the induction of apoptosis and cell cycle arrest. Int J Oncol 53: 835-843, 2018.

9. Chan BC, Ip M, Gong H, Lui SL, See RH, Jolivalt C, Fung KP, Leung PC, Reiner NE and Lau CB: Synergistic effects of diosmetin with erythromycin against ABC transporter over-expressed methicillin-resistant Staphylococcus aureus (MRSA) RN4220/pUL5054 and inhibition of MRSA pyruvate kinase. Phytomedicine 20: 611-614, 2013.

10. Yu G, Wan R, Yin G, Xiong J, Hu Y, Xing M, Cang X, Fan Y, Xiao W, Qiu L, et al: Diosmetin ameliorates the severity of cerulein-induced acute pancreatitis in mice by inhibiting the activation of the nuclear factor- $\kappa \mathrm{B}$. Int $\mathrm{J}$ Clin Exp Pathol 7 : 2133-2142, 2014

11. Yang Y, Gong XB, Huang LG, Wang ZX, Wan RZ, Zhang P, Zhang QY, Chen Z and Zhang BS: Diosmetin exerts anti-oxidative, anti-inflammatory and anti-apoptotic effects to protect against endotoxin-induced acute hepatic failure in mice. Oncotarget 8 : 30723-30733, 2017.

12. Liu B, Shi Y, Peng W, Zhang Q, Liu J, Chen N and Zhu R: Diosmetin induces apoptosis by upregulating p53 via the TGF- $\beta$ signal pathway in HepG2 hepatoma cells. Mol Med Rep 14 159-164, 2016

13. Qiao J, Liu J, Jia K, Li N, Liu B, Zhang Q and Zhu R: Diosmetin triggers cell apoptosis by activation of the p53/Bcl-2 pathway and inactivation of the Notch $3 / \mathrm{NF}-\kappa \mathrm{B}$ pathway in HepG 2 cells. Oncol Lett 12: 5122-5128, 2016.

14. Zhang Y, Jiang Y and Lu D: Diosmetin suppresses neuronal apoptosis and inflammation by modulating the phosphoinositide 3-kinase (PI3K)_AKT_nuclear factor- $\kappa \mathrm{B}(\mathrm{NF}-\kappa \mathrm{B})$ signaling pathway. Med Sci Monit 25: 8, 2019.

15. Glick D, Barth S and Macleod KF: Autophagy: Cellular and molecular mechanisms. J Pathol 221: 3-12, 2010.
16. Nikoletopoulou V, Markaki M, Palikaras K and Tavernarakis N: Crosstalk between apoptosis, necrosis and autophagy. Biochim Biophys Acta 1833: 3448-3459, 2013.

17. Fan YJ and Zong WX: The cellular decision between Apoptosis and autophagy. Chin J Cancer 32: 121-129, 2013.

18. Zhang D, Wang W, Sun X, Xu D, Wang C, Zhang Q, Wang H, Luo W, Chen Y, Chen $\mathrm{H}$ and Liu Z: AMPK regulates autophagy by phosphorylating BECN1 at threonine 388. Autophagy 12: 1447-1459, 2016

19. Mihaylova MM and Shaw RJ: The AMPK signalling pathway coordinates cell growth, autophagy and metabolism. Nat Cell Biol 13: 1016-1023, 2011.

20. Yuan M, Zhang L, You F, Zhou J, Ma Y, Yang F and Tao L: MiR-145-5p regulates hypoxia-induced inflammatory response and apoptosis in cardiomyocytes by targeting CD40. Mol Cell Biochem 431: 123-131, 2017.

21. Zhang J,HeZ,Xiao W,Na Q, Wu T,Su K and Cui X: Overexpression of BAG3 attenuates hypoxia-induced cardiomyocyte apoptosis by inducing autophagy. Cell Physiol Biochem 39: 491-500, 2016.

22. Jia Z, Lin L, Huang S, Zhu Z, Huang W and Huang Z: Inhibition of autophagy by berberine enhances the survival of H9C2 myocytes following hypoxia. Mol Med Rep 16: 1677-1684, 2017.

23. Zhang S, Zhao Y, Xu M, Yu L, Zhao Y, Chen J, Yuan Y, Zheng Q and Niu X: FoxO3a modulates hypoxia stress induced oxidative stress and apoptosis in cardiac microvascular endothelial cells. PLoS One 8: e80342, 2013.

24. $\mathrm{Su}$ Y, Tian H, Wei L, Fu G and Sun T: Integrin $\beta 3$ inhibits hypoxia-induced apoptosis in cardiomyocytes. Acta Biochim Biophys Sin (Shanghai) 50: 658-665, 2018.

25. Kanazawa H, Imoto $K$, Okada $M$ and Yamawaki $H$ : Canstatin inhibits hypoxia-induced apoptosis through activation of integrin/focal adhesion kinase/Akt signaling pathway in $\mathrm{H} 9 \mathrm{c} 2$ cardiomyoblasts. PLoS One 12: e0173051, 2017.

26. Liu Q, Ci X, Wen Z and Peng L: Diosmetin alleviates lipopolysaccharide-induced acute lung injury through activating the Nrf2 pathway and inhibiting the NLRP3 inflammasome. Biomol Ther (Seoul) 26: 157-166, 2018.

27. Zhang Z, Li H, Chen S, Li Y, Cui Z and Ma J: Knockdown of microRNA-122 protects $\mathrm{H} 9 \mathrm{c} 2$ cardiomyocytes from hypoxia-induced apoptosis and promotes autophagy. Med Sci Monit 23: 4284-4290, 2017

28. Sung HK, Chan YK, Han M, Jahng JWS, Song E, Danielson E, Berger T, Mak TW and Sweeney G: Lipocalin-2 (NGAL) attenuates autophagy to exacerbate cardiac apoptosis induced by myocardial ischemia. J Cell Physiol 232: 2125-2134, 2017.

29. Egan DF, Shackelford DB, Mihaylova MM, Gelino S, Kohnz RA, Mair W, Vasquez DS, Joshi A, Gwinn DM, Taylor R, et al: Phosphorylation of ULK1 (hATG1) by AMP-activated protein kinase connects energy sensing to mitophagy. Science 331 : 456-461, 2011.

30. Zhou C, Gu J, Zhang G, Dong D, Yang Q, Chen MB and Xu D: AMPK-autophagy inhibition sensitizes icaritin-induced anticolorectal cancer cell activity. Oncotarget 8: 14736-14747, 2017.

31. Zhang H, Liu B, Li T, Zhu Y, Luo G, Jiang Y, Tang F, Jian Z and Xiao Y: AMPK activation serves a critical role in mitochondria quality control via modulating mitophagy in the heart under chronic hypoxia. Int J Mol Med 41: 69-76, 2018.

32. Xu Z, Yan Y, Xiao L, Dai S, Zeng S, Qian L, Wang L, Yang X, Xiao $Y$ and Gong Z: Radiosensitizing effect of diosmetin on radioresistant lung cancer cells via Akt signaling pathway. PLoS One 12: e0175977, 2017.

33. Ge A, Liu Y, Zeng X, Kong H, Ma Y, Zhang J, Bai F and Huang M: Effect of diosmetin on airway remodeling in a murine model of chronic asthma. Acta Biochim Biophys Sin (Shanghai) 47 604-611, 2015.

34. Yang K, Li WF, Yu JF, Yi C and Huang WF: Diosmetin protects against ischemia/reperfusion-induced acute kidney injury in mice. J Surg Res 214: 69-78, 2017.

This work is licensed under a Creative Commons Attribution-NonCommercial-NoDerivatives 4.0 International (CC BY-NC-ND 4.0) License. 